\title{
Universal Computation in Fluid Neural Networks
}

\author{
RICARD V. SOLÉ \\ Complex Systems Research Group, Departament de Fisica i Enginyeria Nuclear, Universitat Politécnica \\ de Catalunya, Sor Eulàlia d'Anzizu s/n. Campus Nord, Mòdul B4 08034 Barcelona, Spain
}

JORDI DELGADO

Departament de Llenguatges i Sistemes Informatics, Facultat d'Informatica, Universitat Politécnica de Catalunya, Pau Gargallo 5, 08028 Barcelona, Spain

Received July 14, 1995; accepted February 9, 1996

\begin{abstract}
Fluid neural networks can be used as a theoretical framework for a wide range of complex systems as social insects. In this article we show that collective logical gates can be built in such a way that complex computation can be possible by means of the interplay between local interactions and the collective creation of a global field. This is exemplified by a NOR gate. Some general implications for ant societies are outlined. @ 1996 John Wiley \& Sons, Inc.
\end{abstract}

Key Words: Emergent computation, ants, universal computation

\section{INTRODUCTION}

$\mathbf{T}$ he collective capabilities of social insects are known to be largely the result of a nonlinear cooperative phenomenon $[1,2]$. Typically, individual ants display a limited repertoire of activity patterns [2-4]. Unlike individuals, the colony as a whole display very complex patterns, involving search, information transfer and some computational processes. Examples of such computational habilities are task allocation $[5,6]$ or the decision between two different food sources $[7,8]$. In this sense, when looking at an ant colony, we perceive several properties in their organization which are commonly shared with the brain and eventually with standard neural networks (NN). These common properties have been listed in several studies $[1,9,10]$ and are summarized by: robustness of behavioral patterns against noise, collective decision-making and emergent computation.

In ant colonies, a parallel distributed processing of information is performed. Individuals gather information from the environment as well as from their nestmates. As a consequence, a given distribution of tasks is always present. As pointed out by Hölldobler and Wilson [3], much of the structure of the ant colony is based on order parameters, defined as the proportion of individuals existing in one state or another. As they say: "the most striking social effects turn out to be the holistic outcomes of mass communication combined with the rise and decline of pheromones and foodstuffs."

There is an important property that makes insect societies rather different from brains: the system is fluid, i.e., information transfer is gathered by moving entities. The "connection" among individuals is a transient phenomenon, unlike synaptic connections, which are (more or less) fixed. It is well known that in the last case, a neural network can (under some constraints) perform as an associative memory. The power of NN is undiscussed, but how far can go, computationally, those dynamical systems sharing some properties with NN but with no fixed connectivity?

In ant colonies, information transfer is performed by the colony as a whole. It is stored (at least in a short-term), propagated and processed. As a new source of energy or a danger is discovered by an individual, it can be translated to the colony 
and then a collective decision is adopted. But interestingly, this process is not necessarily deterministic: probabilistic behavior of individuals play a very important role [7].

Recent studies on order and chaos in ant colonies [9] have shown that the analogies between neural nets and ant colonies can be sucessfully introduced in a new class of models: the so called fluid neural networks (FNN, see [11]). By using this approach, some phenomena, as coherent global oscillations of colony activity have been fairly well reproduced, in agreement with experimental observations [12, 13]. These phenomena have been theoretically interpreted as computational processes close to phase transition points $[6,11]$.

$\mathbf{T}$ his problem belongs to a class of models concerning computation in distributed systems. Some nonlinear, spatially-extended models have been suggested to be able to perform computation. In particular coupled map lattices (CMLs), a model of spatially extended nonlinear systems, have been explored [14]. It was shown that CMLs are a valid model for a parallel deterministic analog machine. In this context, excitable media would be candidates to computational systems.

More recently, it has been claimed that ant colonies would be, in principle, capable of universal computation [15]. This is a very interesting suggestion and was based on a mathematical model. This model is defined through a set of master equations. Let us consider $N$ ants. If $p_{i}$ is the fraction of ants in state $i$ (with $i=1, \ldots, M$ ), then a set of master equations, i.e.,

$$
\frac{d_{p i}}{d t}=-\sum_{j=1}^{M} A_{i j}(s) p_{i}+\sum_{j=1}^{M} A_{i j}(s) p_{j}+\mathcal{F}\left(B_{i j k}, e_{i j}, p_{j}\right)
$$

was used, where: i) $A_{i j}(s)$ is the set of transition probabilities of switching from task $i$ to task $j$ (for a given environmental state $s$ ) and ii) $B_{i j k}$ is the probability that an ant in state $i$ encounters an ant in state $j$ (with some probability $e_{i j}$ ) and then switch (because of the interaction) to state $k$. The whole set of changes resulting from pair interactions is indicated by the last term.

The model was shown to be able of amplification and oscillations (though not in terms of limit cycles, as it should be in natural dissipative systems). Finally, a NOR gate was constructed. All these results were obtained by considering macroscopic properties defined through the ratio of the ant numbers in different states. Since any boolean circuit can be built up using the NOR gate, universal computation (see below) is in principle possible.

Some of the assumptions of the Lachmann and Sella model [15] are, in our view, quite ad hoc. Each component (the amplifier, the oscillator and the NOR gate) are defined through a different set of nonlinear equations. On the other hand, several equations are of the type $d p_{i} / d t=0$, which makes the underlying assumptions unlikely to translate to real systems.

Here we want to address the problem of how complex the computational capacity of a FNN can be. As a theoretical framework, we consider the idea of universal computation. It will be shown, by means of a simple model, that ant colonies can act as some type of (collective) logic gates and in particular that the NOR gate can easily be constructed in a natural way. In this framework the amplifier and the NOR gate, together with a characteristic temporal scale of response are all obtained into the basic mechanism (see below).

\section{FLUID NEURAL NETWORKS}

A general formalism has been recently proposed for the study of groups of social insects $[6,11]$ as well for collectives of robots or the immune system. This approach has been called fluid neural networks. We consider a set of $N$ entities (which we will call "automata" or "ants") defined, in the general case, by a set of elements each one represented by $m$ bits, i.e., $\mathbf{S}_{i}(t) \equiv\left(S_{i}^{1}(t), \ldots, S_{i}^{m}(t)\right)$. Here we take $S_{i}^{j} \in \sum \equiv\{+1,-1\}$. In previous papers, these entities moved at random on a $L \times L$ grid, and this rule introduces the fluidity on the network, but other ways of random change of connections are allowed. For simplicity, we consider single-bit individuals (i.e., $m=1$ ). The density of ants will be indicated by $\rho=N / L^{2}$. Each time step, all elements will move at random towards one of the eight nearest lattice positions (if available).

The dynamics of this network is described by means of a transition rule defined by the transition probability:

$$
P\left(S_{i} \rightarrow-S_{i}\right)=\frac{1}{2}\left[1-\tanh \left(\beta\left(h_{i}(t) S_{i}(t)-\Theta_{i}\right)\right)\right]
$$

where $h_{i}$ is the local field, defined as:

$$
h_{i}(t)=\sum_{j \in B_{i}(\rho)} J_{i j} S_{j}(t)
$$

being the sum extended over the "neighbors" of $S_{i}(t)$. The neighborhood is indicated by $B_{i}(\rho)$ and here the eight nearest positions are taken (Moore neighborhood). $\Theta_{i}$ is a given threshold, also known as the "external field". Here the strength and type of connection is given by $J_{i j}=\lambda\left(S_{i}(t), S_{j}(t)\right) \in \mathcal{R}$, i.e., a state-dependent connection.

For our study, where $S_{i} \in \sum=\{+1,-1\}$, the connection matrix reduces to the following $2 \times 2$ table:

$$
\Lambda\left[\begin{array}{ll}
\lambda(+,+) & \lambda(+,-) \\
\lambda(-,+) & \lambda(-,-)
\end{array}\right]
$$

At a given time step, the interaction $J_{i j}$ between the $i$ th and the $j$ th elements is equal to $\lambda\left(S_{i}(t), S_{j}(t)\right) \in \mathbf{\Lambda}$ by depending on the current states of the given elements. More precisely, $J_{i j}=\lambda(+,+)$ if $S_{i}=S_{j}=+1, J_{i j}=\lambda(+,-)$ if $S_{i}=+1$ and $S_{j}=-1$ and so on. For our previous model $[6,11]$ (where active and inactive ants were considered) some experimental studies have shown that the sign of the matrix elements can be determined experimentally (B. Cole, personal communication).

For the particular case where $\lambda_{i j}=1$ for all cases (ferromagnetic FNN) it is not difficult to show (Delgado and Solé, un- 
published) that the macroscopic state defined by $m(\{\mathbf{S}\})=\sum$ $S_{j} / N$ evolves following the mean-field equation:

$$
\Gamma \frac{\partial m}{\partial t}=-m+\tanh [\beta \rho(m+h)]
$$

where $h$ is the so called external field and $\Gamma$ is a constant rate, defining the characteristic time scale of relaxation. For $h=0$, the stable attractors of this system will be: i) $m_{0}^{*}=0$ for $J \equiv \beta \rho<0=J_{c}$ and $m_{ \pm}^{*} \neq 0$ (with $m_{+}^{*}=-m_{-}^{*}$ ) for $J>J_{c}$. We say that symmetry breaking occurs at $J_{c}=0$, where two new states emerge. In terms of information, we can say that the original information is doubled because two new attractors become available [16]. These attractors can be visualized as the minima of a free-energy $\Phi(m, h)$ of the system, i.e.,

$$
\frac{\partial \Phi\left(m^{*}, h\right)}{\partial m^{*}}=0
$$

One possible free-energy function (also called GinzburgLandau potential) compatible with the previous equation for $m(t)$ is:

$$
\frac{1}{N} \Phi(m, h) \equiv \phi(m, h)=\frac{\beta \rho}{2} m^{2}+\rho \ln \{\cosh [\beta(p m+h)]\}
$$

in such a way that our dynamics is defined by a gradient system, i.e.:

$$
\Gamma \frac{\delta m}{\delta t}=-\frac{\partial \Phi(m, h)}{\partial m}
$$

and so a relaxation towards $m_{+}^{*}$ or $m_{-}^{*}$ (depending on the initial state $m(0))$ will occur.

We have introduced this particular case because it illustrates the early suggestions by Haken [16, 17] about information processing in complex systems. To store information, the system has to be able to stabilize the attractors in deep enough minima (i.e., those defined by $\Phi(m, h))$. But in order to process information, switching among attractors is necessary. If, through some self-regulated mechanism, a switch among attractors is available, processing becomes possible. Though it has been suggested that complex computation takes place in systems poised at critical points, here we suggest a different strategy. The system can store information by means of attractors and switch among them by moving through critical points.

\section{TASK ALLOCATION AND GLOBAL ATTRACTORS}

A particularly relevant observation about ant colonies (and relevant in our study) is the existence of a distribution of tasks in the colony. In this sense, some given numbers of individuals engaged in different activities are observed. These numbers can be determined by genetic constraints, linked with morphological traits [2,3] or, more frequently, be the result of a self-organizing pattern emerging from the local interaction among individuals. In this sense, the final distribution of tasks will be an "attractor" of the ant colony dynamics [10].

Following this observation, we can consider several particular situations described by our FNN model. By an adequate choice of the connectivity matrix, we can reach a particular global state by means of local interactions. Assuming that $J>J_{c}$ then the matrix

$$
\Lambda_{F}=\left(\begin{array}{ll}
1 & 1 \\
1 & 1
\end{array}\right)
$$

which defines the ferromagnetic FNN, is in fact the discrete counterpart of the previous mean-field model (see Section 2). For $\beta=0$, half of the automata will be in state +1 and half in -1 . When $\beta>0$, symmetry breaking takes place. One of the possible attractors $\left\{m_{+}^{*}, m_{-}^{*}\right\}$ will be chosen.

Another possibility in order to reach a particular attractor can be to provide an external field $h>0$. Then an assymetry is introduced from the beginning and the system will be in one of the global states $m_{+}^{*}$ or $m_{-}^{*}$. But there is an additional possibility. We take $\beta>0$ and an appropiate $\Lambda$ matrix such that transitions towards a particular state are more likely to occur. As an example, we can take:

$$
\Lambda_{F_{\varnothing}}=\left(\begin{array}{ll}
1 & 1-\varepsilon \\
1 & 1-\varepsilon
\end{array}\right)
$$

(where $\varepsilon \in(0,1)$ ) is such that leads to a FNN where almost all elements move towards the state $S_{i}=+1$, and so $m \rightarrow m_{+}^{*}$. In this way, we can store information. This is just a particular case, but is easily extended to FNN with several tasks [10].

N ow the basic problem in order to perform computation is to have a mechanism of switching among attractors. In this way, we process information. One possibility, as stressed by Haken $[16,17]$, is to be able to switch by means of fluctuations, which are larger as we approach $J=J_{c}$, the critical point where fluctuations diverge. But in this state the reliability of the system is lowered precisely due to fluctuations. So what we need is an additional mechanism which should be modified by the own system (say the ants), and able to reach different attractors under suitable inputs. This mechanism, chemical communication, is discussed in the next section.

\section{CHEMICAL COMMUNICATION}

The previous mechanism of communication, which involves direct contact among neighbors, is just part of the story. Chemical communication in ants play a very important role and will be also considered in our study. Some general comments are of interest:

a) Ants interact among themselves both by direct contact (being contact rates very important in colony organization, see Gordon, [18]) and by means of chemical substances [2].

b) By means of both phenomena, the ant behavior can switch from the current state (task) to another. In any case, the ant can respond to the chemical signal and reinforce it. As a consequence, there is a nontrivial global behavior sustained 
by means of the individual activity of ants, being this activity simultaneously modified by the chemical field. This is an emergent phenomenon, and is represented in Figure 1, following [16]. The individual ants and the global activity pattern are related in two directions: from top to bottom and from bottom to top.

c) Computation takes place collectively. The ant colony perceive external signals and monitors its environment, being then able to make decisions. These decisions result from different inputs which can be conflicting (two different food sources, enemies, etc.). Local inputs must be processed collectively in order to obtain an adequate global output. As stated by Wilson: "(local information) is judged principally, and perhaps exclusively, by the "electorate" response of the colony through all-or-none "voting" by the individual ants" [2].

d) The ant colony needs to be flexible. Once a given information is detected, chemical communication (as trails) makes possible the global response. The stability of the chemical signal is thus relevant for colony behavior. But after a given source of information is gone, the colony has to be able to switch again towards the "normal" state, defined by means of some task distribution. Again by Wilson: "the level of accuracy (of chemical communication) has been arrived at a compromise between the utmost effort of the ant's chemosensory apparatus to follow trails accurately and, simultaneously, the need to reduce the quantity and increase the volatility of the trail substance in order to minimize overcompensation in the mass response" [2].

The last two comments can be well represented by means of a simple diagram, which uses the idea of attractors, as shown in Figure 2 (see also Ref. [10]). We can imagine the standard distribution of tasks as a given set of numbers of indi- viduals engaged in different activities [10]. This distribution is, in our approach, an "attractor" of the dynamical state. In Figure 2(a), it is shown by an energy landscape (defined for example in [10], based on the standard approach to neural nets) where the minimum represents the most probable distribution of states. Here $\alpha$ is the deepest valley, defining the most probable distribution, though may be other small valleys $(\beta)$ could be present. As an external signal is detected, the system should be able to switch towards other attractors (i.e., $\alpha \rightarrow \beta$, by changing the landscape) in order to perform new tasks [19].

In our study, we consider a given chemical concentration $C(i, j)$ which can be detected and reinforced by the ants. This substance, in absence of ants, will have a simple dynamical evolution given by the diffusion equation (DE):

$$
\frac{\partial C}{\partial t}=-\mu C+D \nabla^{2} C
$$

Here $\mu$ stands for the spontaneous rate of decay, $D$ is the diffusion coefficient, and

$$
\nabla^{2}=\partial_{x}^{2}+\partial_{y}^{2}
$$

is the two-dimensional Laplace operator. A discretization of the previous DE will be used, where we will use the following numerical approximations:

$$
\begin{aligned}
& \nabla^{2} C(i, j, t)= \\
& \frac{C(i+1, j, t)+C(i-1, j, t)+C(i, j+1, t)+C(i, j-1, t)-4 C(i, j, t)}{\delta x^{2}}
\end{aligned}
$$

where we take $\delta x=1$, and time is also discretized as:

$$
\frac{\partial}{\partial t} C\left(i, j, t_{k}\right)=\frac{C\left(i, j, t_{k-1}\right)-C\left(i, j, t_{k}\right)}{\delta t}
$$

FIQURE 1

\section{Collective Behavior}
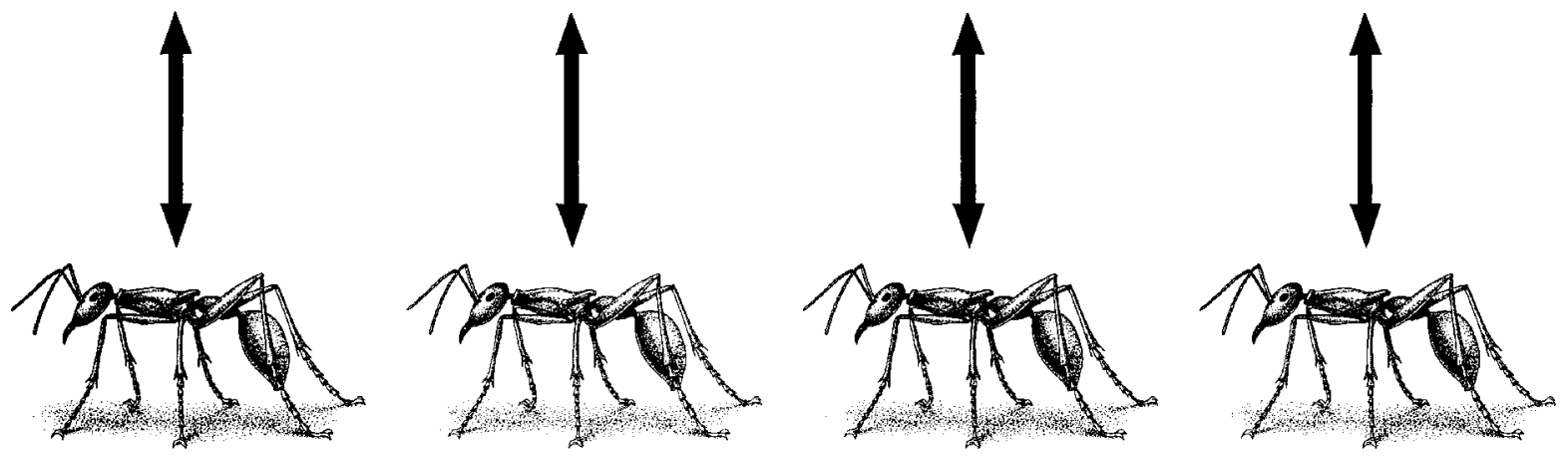

Emergent computation in social insects. Ants interact among them through direct contact and through chemical fields. Then the global behavior (the activity pattern) acts over the single ants as an order parameter. These emergent patterns cannot be reduced to the sum of the activities of the basic units (the ants). 
where $1 \leq i, j \leq L$ and $t_{k}=k \delta t$ (here we use $\delta=10^{-3}$ ). Clearly, our numerical calculation of dynamics of the chemical component defines two distinct time scales for individuals and the chemical field.

If ants are present. a new term should be included in the previous PDE, involving the reinforcement of the chemical signal, as will be done in the following section. It is interesting to mention here that chemical communication, together with tactile stimuli, is able to provide strong cohesiveness even to large societies. Army ants, which are among the largest societies, show spatially self-organized swarm raids that cover $1000 \mathrm{~m}^{2}$ in a day [4]. It is one of the best examples of collective decision making without centralized control of any kind. In this context, a remarkable study by Mikhailov [20] on mass communication in distributed systems has been performed, involving the formal approach of neural networks together with chemical mediators.

\section{UNIVERSAL COMPUTATION}

Spatially extended dynamical systems can be complex enough to show interesting computational properties [14]. The possibility of universal computation [21] can be proved in two ways. One possibility is the direct way, i.e., showing that an Universal Turing Machine (UTM) can be simulated by the system (see for example the approach by Holden et al. [14]). The indirect way consists in showing that the system can emulate a device with which a UTM can be constructed. The last approximation has been used in showing that some cellular automata models, as the Game of Life, can perform universal computation [22]. In particular, if a NOR gate can be built, then any other logic gate can be obtained by an adequate connection of coupled NOR gates.

In this section, we show how a NOR collective gate can be obtained in a simple way. First, we have to define the "normal" state of the network, which can be understood as a given distribution of tasks. For simplicity, we will define a colony state where the elements in our FNN are mainly in state $S_{i}=+1$. This can be achieved in several ways. Three situations are considered in the following subsections.

\subsection{Matrix $\Lambda_{0}$, Chemical Switch}

This is the simplest collective NOR gate. Let us remind that we want to obtain the following table:

\begin{tabular}{ccc}
\hline Input $1\left(I_{1}\right)$ & Input 2 $\left(I_{2}\right)$ & $\Omega$ \\
\hline 0 & 0 & 1 \\
1 & 0 & 0 \\
0 & 1 & 0 \\
1 & 1 & 0 \\
\hline
\end{tabular}

Where a properly defined "collective" response $\Omega$ needs to be introduced. Here we take $\beta>0$ and the connectivity matrix will be $\Lambda_{0}$, as defined in (8b). As a consequence of this particular choice, a given task distribution will be obtained, being the $S_{i}=+1$ individuals the most abundant.

Now let us define the two external "inputs," $I_{1}$ and $I_{2}$, necessary in order to construct the logical gate. Following our previous discussion, they can be two signals placed at two different points of the lattice (the environment). Here we use two opposite vertex. As a signal, we take a given fixed concentration $C_{0}$ which can $\left(I_{i}=1\right)$ or cannot $\left(I_{i}=0\right)$ be present. At a given time step (and for some time $\tau$ ) we fix the state ${ }^{1}$ of both points to $C_{0}$. Then if a given ant detects, at a given lattice point, a concentration $C>\theta$, where $\theta$ is a threshold, it reinforces the local concentration by an amount $\psi$. The local concentration acts on the local field in the following way:

$$
h_{i}\left(t, C_{i}\right)=\sum_{j \in B_{i}(\rho)} J_{i j} S_{j}(t)-C_{i}
$$

here $C_{i}$ is the local concentration perceived by the single ant (located at a given lattice point). We see that in this way, the local field is modified, and the new transition probability makes possible to switch towards a different state. Physiologically, this is nothing but a change in the excitability of the individual ants. As $C_{i}$ grows, it becomes more and more likely to switch towards $S_{i}=-1$. If the self-reinforcement of the chemical field is strong enough (and this will depend specially on $\mu$ and $\rho$ ) the whole system can switch. The local signals have been amplified and a new attractor has been created (as it was shown in Figure 2). In Figure 3 we show, for a particular set of parameters, the evolution of the three main quantities defined in our study: $m, C$ and $\Omega$. Here $\Omega$ is the discrete output obtained from our collective computation, and it is defined as 

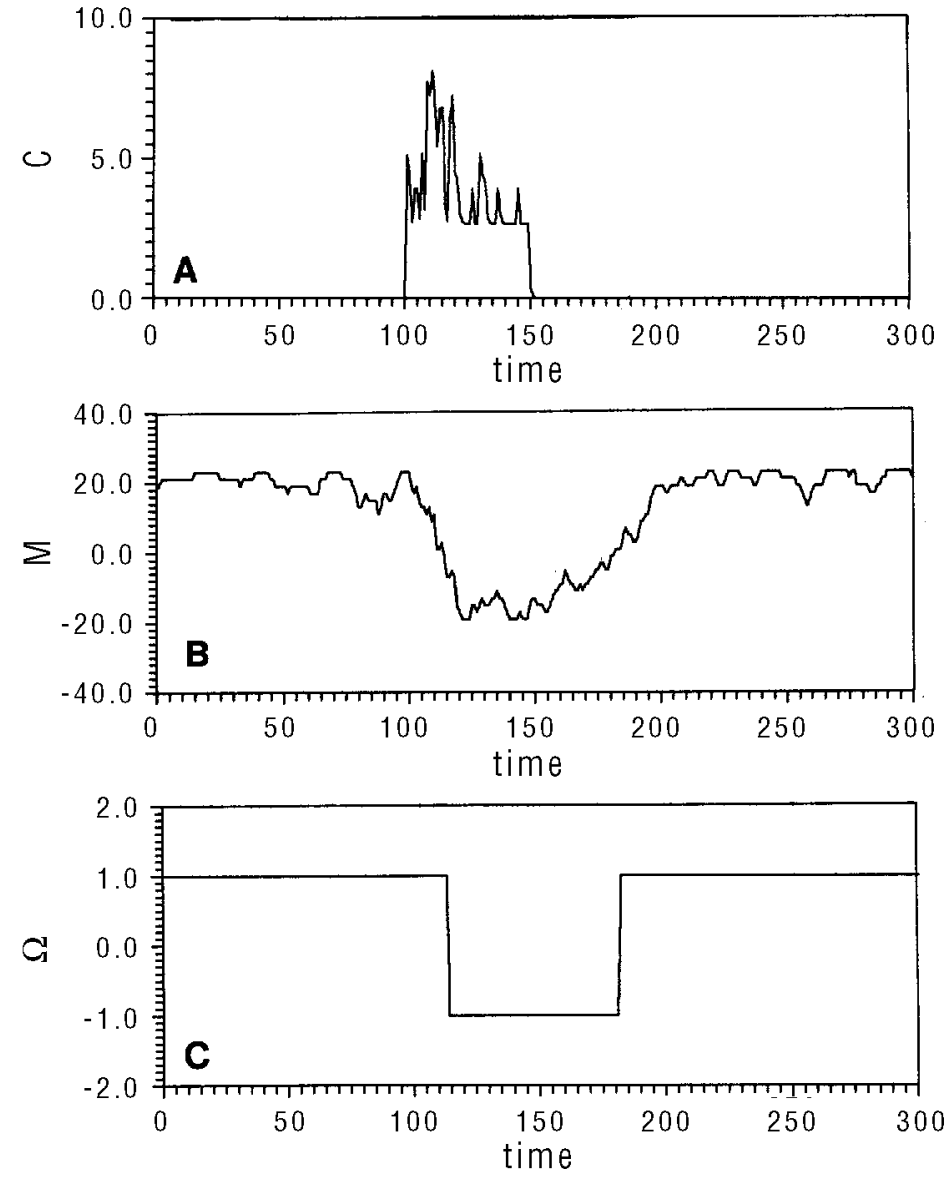

Collective NOR gate. An example of the dynamics of the three basic components of our model is shown (see text). As an external signal is introduced (from step 100 to 150) a chemical field is formed, and a switch is obtained. For this parameter combination, the switch is a transient situation. After some time steps, once the chemical field decay to zero, the previous state is recovered.

$$
\Omega=H\left[\sum_{j=1}^{N} S_{i}(t)\right] .
$$

Where $H(z)$ is the Heaviside step function, defined as $H(z)=1$ if $z>0$ and $H(z)=0$ otherwise. We can see that after the appearance of the external signal, the system is able to switch towards the negative values and so $\Omega=0$. In other cases (see below) the self-reinforcement of the chemical field is strong enough that the new state is maintained indefinitely. For very slow densities, both fluctuations and small information transfer makes the gate very unstable.

\subsection{Matrix $\Lambda_{F}$, Nonzero Threshold}

A different possibility is a FNN with ferromagnetic interactions (as defined by the matrix $\Lambda_{F}$ ) and where a given nonzero threshold $\Theta$ is introduced. Again, ants will move towards $m_{+}{ }^{*}$ (here we take $\beta>0$ ). Now the local field is given by:

$$
h_{i}\left(t, C_{i}\right)=\sum_{j \in B_{i}(\rho)} J_{i j} S_{j}(t)-\Theta+C_{i}
$$

and the previous rules are the same as before. For an adequate choice of parameters, a similar scenario is obtained. This can also be obtained using a different chemical field $C_{i}^{*}$, instead of a fixed threshold, $\Theta$. If no external input is present, the ants can self-maintain this new field. When the external signal is detected, the new field would be more reinforced, and the transition can take place.

\subsection{Matrix $\Lambda_{F}$, Two Chemicals}

This is a more sophisticated situation. The system starts with a small $\beta>0$ value and the $\Lambda_{F}$ matrix. If no external inputs are present, both states $S_{i}= \pm 1$ are equally likely to occur. Now let us assume that two different types of inputs (which we can arbitrarily call "0" and " 1 ") are allowed, with concentrations $C^{(1)}$ and $C^{(2)}$. So now $I_{i}$ $=0$ means that $C^{(1)}$ is being introduced as an input, and $I_{i}=1$ means that $C^{(2)}$ is used. Then two different chemical substances are specified as a symbol of the input alphabet. Both chemicals will have characteristic rates of decay $\left(\mu_{1}, \mu_{2}\right)$ and diffusion rates $\left(D_{1}, D_{2}\right)$.

Now the local field will read:

$$
h_{i}\left(t, C_{i}^{(1)}, C_{i}^{(2)}\right)=\sum_{j \in B_{i}(\rho)} J_{i j} S_{j}(t)-C_{i}^{(1)}+C_{i}^{(2)} .
$$

Where each chemical is acting, as we see, in opposite ways. Again, as ants find locally any of the chemical signals, they reinforce the local values (if $C_{i}^{(k)}>\theta_{i}^{(k)}$, as before). The competition between both chemicals can result in different global outputs. If the specific rate constants are suitably chosen (for example, if $\mu_{1}>\mu_{2}$ and $D_{1}=D_{2}$ ) a NOR gate is obtained.

\subsection{Parameter space}

We have now to see how robust are these collective gates. Their behavior depends upon the parameters involved, and here we analyse the first type of gate. The following constants are used: $C_{0}=10, D=0.2, \theta=10^{-4}$ and $\psi=0.5$. Using a $L=20$ lattice, with $\varepsilon=0.35$ and $\beta=2$, a detailed study of the parameter space $(\rho, \mu)$ has been performed. For each sample, we have discarded $T=200$ transients in order to reach the colony attractor given by matrix $\Lambda_{0}$ and the external signals are introduced over a short period of time ( $\tau=50$ time steps). Then we study whether or not the colony is able to switch towards the new attractor and move back some time later. We take as correct those samples where the colony is able to switch and return to the initial distribution (here $m_{+}^{*}$ ) into a period of 150 time steps.

The phase space of our system, for the previous parameters, is shown in Figure 4. Three regions are observed. The first one is the so called random network (RN, shaded area) 
obtained at very low densities of ants, is a domain where the NOR gate cannot be built. Either random effects become dominant (and the FNN is switching randomly) or only if the two sources are present the system can switch. The dotted area shows a domain where the NOR gate works, but no turning back to the initial attractor is possible. The amplification of the incoming signals is too strong and becomes self-sustained. The white area is the most interesting: the NOR gate is built and the system is flexible enough to move back to the previous attractor. This happens at intermediate densities. As far as the collective gate can switch back to the previous state in a bounded interval of time, a characteristic time scale for computation can be defined. The main properties of this parameter space (as the separation in three well defined areas) are also obtained from the other implementations of the NOR gate.

\section{DISCUSSION}

In this article we have analysed a particular problem involving the computational capacity of FNN. The underlying goal is to explore the computational abilities of groups of social insects, such as ant colonies. As a starting point we have considered the possibility of building a NOR collective gate. A simplified situation was considered, where two external signals $\left\{I_{1}, I_{2}\right\}$ of some type are used, and a binary variable is assigned to them. A binary variable is also defined over the colony states in such a way that we can clearly construct a table for the gate.

The external signals are appropiately amplified by the ants, and the self-reinforced field acts on the individual ant states through a change in the transition probabilities. By depending on the density of ants and the rate of decay of the chemical field, the external inputs can be amplified.Then an emergent pattern is obtained: a self-sustained chemical field is created. As a consequence a global colony organization is reached. Some parameter combinations $(\rho, \mu)$ makes the system more or less flexible, eventually switching back towards other attractors as the external inputs are removed. Following this idea, we have obtained a NOR gate in several ways. One or two chemicals can be used. They can act on all types of ants or in different ways for different states.

The existence of a domain of densities $(\rho)$ where maximum flexibility is allowed is consistent with other experimental and theoretical works involving networks of patrolling ants [23]. The encounter rates among ants has been shown to be of extreme importance as an organizing factor for the colony behavior. In terms of dynamics, a low density makes possible to enhance fluctuations and switching among attractors. If the density is high enough, we can also ensure (at least transiently) the stability of the attractors. This compromise has also been observed in other situations [6, 11].

This work can be extended to more general situations. If an arbitrary set of chemical signals $\left\{C_{i}^{(k)}\right\}$ is involved (here $k=1,2, \ldots, f)$ then the local field $h_{i}$ perceived by the individual ants will be:

$$
h_{i}\left(t,\left\{C_{i}^{(k)}\right\}\right)=\sum_{j \in B_{i}(\rho)} J_{i j} S_{j}(t) \sum_{k=1}^{f} \eta^{(k)} C_{i}^{(k)}
$$

where $\eta^{(k)} \in\{-1,+1\}$ by depending on how each field behaves. The previous results would be then generalized. Equation (12) contains much of the ingredients of ant colony dynamics. Antto-ant interactions and the creation and interaction through chemical fields are both involved. The importance of each term will be different for different species, but their sensitivity to fluctuations and how the colony behavior is tuned are rather general problems. The appropriate transfer and processing of information requires parameter combinations not too far from phase transition points. In fact, recent theoretical studies show that such points would play a prominent role in the evolution of social behavior [24], as early suggested by Wilson [3].

Two final, particular comments have to be considered for future studies:

a) The FNN model is robust against noise. Though some deterministic cellular automata models (as the Game of Life) have been shown to be able to support universal computation [25] they are not robust when noise is present (as it happens in natural conditions). A random change in a single automaton state can destroy the gate. A FNN finds the robustness through the amplification of incoming information and is only

\section{FIGURE 4}

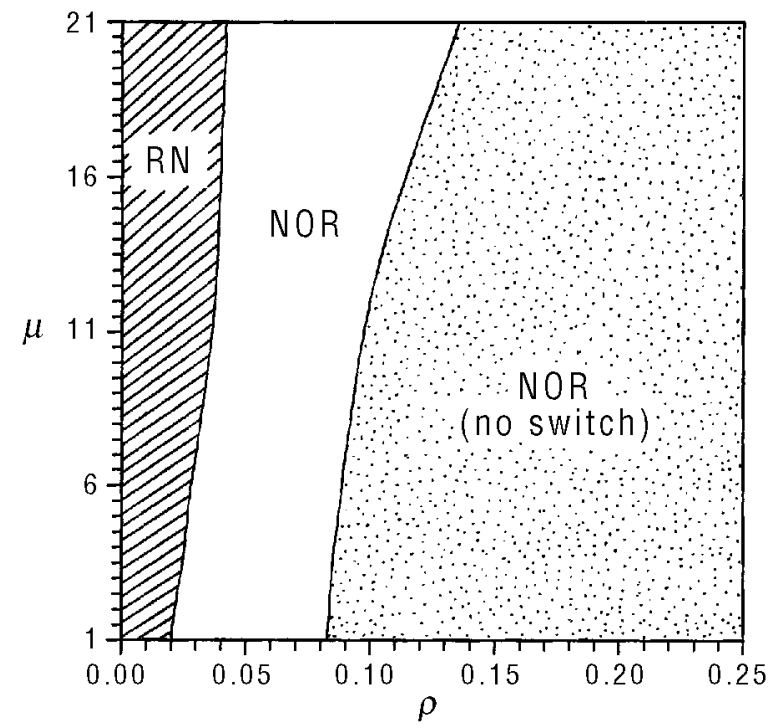

Phase space for the collective NOR gate (see text). Three qualitative domains are obtained. The dashed area (RN) is linked with those parameter combinations unable to define a NOR gate: random fluctuations are dominant. The dotted area is linked with a properly defined NOR gate, but the self-reinforcement of the chemical field is too strong (see text). The white area corresponds to a properly defined NOR gate, and the system returns to the starting attractor once external signals are removed. 
weakly dependent on failure of single units.

b) The FNN (like an ant colony) is a spatially-distributed system. As a consequence, if different inputs enter to the FNN at different spatial locations, competition and pattern formation can occur. We can easily build up other types of collective gates in response to different types of inputs. It will be of interest to know how such, perhaps conflicting, inputs are processed into the system and how complex these computations would be. This also opens new problems. Though the NOR gate has been shown to be constructable, how several gates could be coupled in order to simulate more complex gates is far from trivial.

\section{ACKNOWLEDGMENTS}

The authors would like to thank Ton Sales and Brian Goodwin for several comments and suggestions. Thanks also to D. Gordon, B. Luque, R. Gavaldó, X. Messeguer, and Q. Gabarró. One of us (RVS) is also indebted to Nigel Franks and his colleagues for useful discussions at the Bath University. This work has been partially supported by a grant of UPC PR9407 and DGYCIT PB94-1195. J. Delgado receives support from a grant FI93/3008.

\section{NOTE}

1. Many other possibilities are allowed, in such a way that the input signals are not coupled with the chemical field. For example, if an ant detects an input $I_{i}=1$, it leaves a given amount of chemical. These possibilities have also been explored, leading to similar results.

\section{REFERENCES}

1. E. Bonabeau and G. Theraulaz: Intelligence collective. Hermes, Paris, 1995.

2. E. O. Wilson: The insect societies. Harvard University Press, Cambridge, 1971.

3. B. Hölldobler and E. O. Wilson: The ants. Springer, Berlin, 1990.

4. J. L. Deneubourg, S. Goss, N. Franks, and J. M. Pasteels: The blind leading the blind: modeling chemically mediated army ant raid patterns. J. Insect Behav. 2: pp. 719-725, 1982.

5 M. J. Hatcher, C. Tofts, and N. R. Franks: Mutual exclusion as a mechanism for information exchange within ant nests. Naturwiss. 79: pp. 32-34, 1992.

6. J. Delgado and R. V. Solé: Evidence of a noise-induced transition in fluid neural networks. Physica D (to appear)

7. J. L. Deneubourg, S. Aron, S. Goss , J. M. Pasteels, and G. Duerinck: Random behavior, amplification processes and number of participants: how they contribute to the foraging properties of ants. Physica D22: p. 176, 1986.

8. M. M. Millonas: A connectionist-type model of self-organized foraging and emerging behavior in ant swarms, J. Theor. Biol. 159: p. 529, 1992.

9. R. V. Solé, O. Miramontes, and B. C. Goodwin: Oscillations and chaos in ant societies. J. Theor. Biol. 161: p. 343, 1993.

10. D. M. Gordon, B. C. Goodwin, and L. E. H. Trainor: A parallel distributed model of the behavior of ant colonies. J. Theor. Biol. 156: pp. 293-307, 1992.

11. R. V. Solé and O. Miramontes: Information at the edge of chaos in fluid neural networks. Physica D80: pp. 171-180, 1995.

12. N. R. Franks, S. Bryant, R. Griffiths, and L. Hemerik: Synchronization of the behavior within the nests of the ant Leptothorax acervorum. Bull. Math. Biol. 52: pp. 597-612, 1990.

13. B. Cole: Is animal behavior chaotic? Evidence from the activity of ants. Proc. R. Soc. Lond. B244: pp. 253-259, 1991.

14. A. V. Holden, J. V. Tucker, H. Zhang, and M. J. Poole: Coupled map lattices as computational systems. Chaos 2: pp. 367-376, 1992.

15. M. Lachman and G. Sella: Universal computation in an ant colony: the power of systems with many simple elements. Proceedings of ECAL'95

16. H. Haken: Synergetics, an introduction. Springer, Berlin, 1977.

17. H. Haken: Information and Selforganization. In: Springer Series in Synergetics, vol. 40. Springer-Verlag, Berlin, 1988.

18. D. M. Gordon, R. E. Paul, and K. Thorpe: What is the function of encounter patterns in ant colonies? Anim. Behav. 45: pp. 1083-1100, 1993.

19. D. M. Gordon: Dynamics of task switching in harvester ants. Anim. Behav. 38: pp. 194-204, 1988.

20. A. Mikhailov: Collective dynamics in models of communicating populations. In: Interdisciplinary approaches to nonlinear complex systems, H. Haken and A. Mikhailov (Eds.) Springer Series in Synergetics, vol. 62, pp. 89-108, 1993.

21. J. E. Hopcroft and J. D. Ullman: Introduction to automata theory, languages and computation. Addison-Wesley, Reading, MA, 1979.

22. E. R. Berlekamp, J. H. Conway, and R. K. Guy: Winning ways for your mathematical plays, vol. 2, Academic Press, London, 1985.

23. F. R. Adler and D. M. Gordon: Information collection and spread by networks of patrolling ants. Am. Nat. 140: pp. 373-400, 1992.

24. R. V. Solé and J. Delgado: Phase transitions and the origins of eusociality. In preparation.

25. C. G. Langton: Life at the edge of chaos. In: Artificial life II. SFI Studies in the Sciences of Complexity, vol. X, Addison-Wesley, Reading, MA, 1991. 